\title{
Profile of Hypernatremia in a Tertiary care hospital : A review of 175 cases over a 5 year period in a South Indian population
}

\author{
George A. ${ }^{1}$, Alexander R. ${ }^{2}$, Abraham S.T. ${ }^{3}$, Rhutu Venugopal E V. ${ }^{4}$ \\ ${ }^{1}$ Dr. Alexander George, Consultant, Plastic Surgeon, Department of Plastic Surgery, KIMS Hospital, Cochin, Kerala, \\ India, ${ }^{2}$ Dr.Reena Alexander, Assistant Professor, Department of Physiology, Sree Narayana Institute of Medical Sciences, \\ Ernakulum, India, ${ }^{3}$ Dr. Solomon T Abraham, Past Medical Officer, KIMS Hospital, Cochin, Kerala, India, ${ }^{4}$ Dr. Rhutu \\ Venugopal E V., Past Medical Officer, KIMS Hospital, Cochin, Kerala, India.
}

Corresponding Author: Dr. Reena Alexander, MBBS, Assistant Professor, Department of Physiology, Sree Narayana Institute of Medical Sciences, Ernakulum, Kerala. India. Email: drreenaalexander@gmail.com

\begin{abstract}
Objective: The aim of this study was to ascertain the profile of hypernatremia with an emphasis on the prevalence, development, progress and clinical outcomes in hospitalized patients. Materials and Methods: This study was carried out at a tertiary care centre in south India from March 2011 to march 2016 and included 175 consecutive hospitalized patients who developed hypernatremia during the course of their management. Results: A total of 5558 patients were admitted as in-patients of which 175 patients developed hypernatremia resulting in a prevalence rate of $3.14 \%$. The majority of patients were males $-130(74.28 \%)$ and $57(32.55 \%)$ of the hypernatremic patients were geriatric. The most common disease condition of patients who developed hypernatremia was head injury-37(21.1\%) followed by respiratory disease-23(13.1\%), sepsis-17(9.7\%) and transient hypernatremia-17(9.7\%). 21 patients (12\%) ended in mortality while the rest $154(88 \%)$ recovered and were discharged from hospital. Patients in the mortality group were found to have higher levels of hypernatremia average- 162 when compared to those that survived $151(\mathrm{p}<0.05)$. Conclusion: While there are few reports devoted to hypernatremia in the Indian population, a standardized management protocol needs to be followed and physicians need to focus more on identifying patients who are likely to develop hypernatremia and take step to avoid it by pre-tagging and using preventive measures. This is probably one of the first studies of its kind in a south Indian population and could be an eye opener in the management of hypernatremic patients.
\end{abstract}

Key words: Serum sodium, Hypernatremia, Tertiary care hospital, Head injury, Mortality

\section{Introduction}

Hypernatremia is a hyperosmolar condition resulting from a decrease in total body water relative to the electrolyte content; levels of serum sodium over $145 \mathrm{meq} / \mathrm{L}$ are generally considered as hypernatremia. However if these sodium levels are uncorrected, it can be lethal as it leads to neuronal cell shrinkage and resultant brain injury besides other injuries.

This cascading effect if unchecked will end in higher morbidity and mortality, a fact that has been confirmed by multiple authors [1,2]. Kumar et al in a study on Indian patients, reports that though hypernatremia was less common than other dyselectrolytemia in hospital admission, it was associated with higher mortality [3]. Hypernatremia can occur from diverse etiologies

Manuscript received: $25^{\text {th }}$ February 2018

Reviewed: $6^{\text {th }}$ March 2018

Author Corrected: $15^{\text {th }}$ March 2018

Accepted for Publication: $21^{\text {st }}$ March 2018 including pure water loss, antidiuretic hormone (ADH) deficiency, sweating, diuresis, exogenous salt, vomiting etc., and is a relatively common dyselectrolytemia encountered in hospitalized patients, especially those in the critical care units with mortality ranging from 30$50 \%$ in hospital acquired hypernatremia $[4,5]$ While the clinical manifestations of hypernatremia in humans are well documented [6], even rats with experimentally induced severe hypernatremia were shown to have symmetrical demyelinating lesions in thalami, basal ganglia, cerebral cortex, and hippocampi [7]. In humans neurological manifestations of hypernatremia consist of varying degrees of encephalopathy, seizures, rhabdomyolysis, and rarely subdural hematoma [4].

Geriatric patients are especially prone to develop hypernatremia and studies on the kidney have shown that with aging, renal concentrating mechanisms are 


\section{Original Research Article}

deranged and the longest nephrons which are most critical for concentrating urine get depleted [8]. Studies on the Indian population on hypernatremia are sparse. This retrospective study is probably the first of its kind in a south Indian population carried out in a tertiary care center; with an aim to establish its prevalence, etiology and management and compare it with other centers.

\section{Materials and Methods}

Place of study and type of study- This is a retrospective study of the incidence, cause, disease relationship and management of hypernatremia in hospitalized patients that was carried out in a tertiary care hospital, Cochin, India, over a five year period from March, 2011 to March, 2016.

Sampling methods- All patients who were admitted to the hospital for treatment of any disease condition and found to develop serum sodium levels more than $145 \mathrm{meq} / \mathrm{L}$ were included in the study group and investigated as per protocol.

Sample collection-Patient's age, sex, disease condition, onset and duration of hypernatremia, management of hypernatremia and treatment outcomes were noted. All data for this study group of patients were derived from the hospital computerized medical records and files from the medical record department. All patients were followed up till the time of their discharge from the hospital or death. The demographic, clinical data, disease diagnosis, laboratory data, associated comorbid conditions and management protocols of these patients were studied in detail and then analyzed. Serum sodium levels as well as other associated abnormal laboratory parameters were studied.

Management strategies as well treatments with respect to drug administration that may add to hypernatremia and hypertonic solution administration were recorded. Changes in the serum sodium levels, the clinical condition and management of these patients were followed up till the time of discharge or death.

Inclusion criteria- All in-patients with serum sodium more than $145 \mathrm{meq} / \mathrm{L}$ during the study period were included in this study.

Exclusion criteria- Patients treated on outpatient basis as well as those under observation for less than a day's period were not included in the study.

Statistical analysis- Data entry and analysis were carried out using Microsoft excel and SPSS software packages. Discrete variables were presented as counts and percentages. To find the significance of difference between two proportions, the 'Fisher's exact t test' was applied, $\mathrm{p}$ values $<0.05$ was considered statistically significant.

\section{Results}

Over a five year period, 5558 patients with varying disease conditions were admitted for in-patient management. Of these, 175 patients developed hypernatremia and were included in the study group showing a prevalence rate of $3.14 \%$. The subjects included 130 males (74.28\%) and 45 females $(25.72 \%)$ with a male to female ratio of 3:1. The mean age of hypernatremic patients was 53.02 years (range 8-99).

While the average age of males was 51.96 years (range 21-87), the average age of females was 54.08 years (range 8 months-99 years). Out of the total 175 patients $3(1.7 \%)$ were pediatric and $57(32.55 \%)$ geriatric with the geriatric group forming almost a third of the study group. There were $17(9.7 \%)$ patients with transient hypernatremia, while the rest 158 had persistent hypernatremia $(90.2 \%)$.

In our study, the most common disease condition of patients who developed hypernatremia was head injury-37(21.1\%) followed by respiratory disease-23(13.14\%), sepsis-17(9.7\%), transient hypernatremia-17(9.7\%), fever with diarrhoea$16(9.1 \%)$ renal disease-15(8.57\%), neurological disease-13(7.4\%), psychiatric diseases with drug and substance abuse$10(5.7 \%)$, uncontrolled diabetes mellitus-10(5.7\%), cardiac disease and hypertension- $9(5.1 \%)$, hypothyroidism- $6(3.4 \%)$ and electrocution-2(1.1\%).

Death occurred in 21(12\%) cases out of which $8(4.5 \%)$ were due to traumatic brain injury. 4 patients of head injury with hypernatremia were diagnosed to have diabetes insipidus. 74(42.2\%) of patients were in intensive care units when the hypernatremia was detected. The duration of hypernatremia varied from 2 day to 12 days.

Hypernatremia levels in patients who ended in mortality- $162 \mathrm{meq} / \mathrm{L}(\mathrm{n}=21)$ were significantly higher than those that survived-151meq/L $(\mathrm{n}=154)(\mathrm{p}<0.05)$. 
Original Research Article

Table-1: Disease condition associated with hypernatremia.

\begin{tabular}{|ll|ll|}
\hline 1. & Disease condition & 2. & Number of patients \\
\hline 3. & Head injury & 4. & $37(21.1 \%)$ \\
\hline 5. & Respiratory disease & 6. & $23(13.1 \%)$ \\
\hline 7. & Sepsis & 8. & $17(9.7 \%)$ \\
\hline 9. & Transient hypernatremia & 10. & $17(9.7 \%)$ \\
\hline 11. & Diarrhea and fever & 12. & $16(9.1 \%)$ \\
\hline 13. & Renal & 14. & $15(8.5 \%)$ \\
\hline 15. & Neurological disease & 16. & $13(21.1 \%)$ \\
\hline 17. & Uncontrolled Diabetes mellitus, & 18. & $10(5.7 \%)$ \\
\hline 19. & Psychiatric disease with Drugs and substance abuse & 20. & $10(5.7 \%)$ \\
\hline 21. & Cardiac disease and Hypertension & 22. & $9(5.1 \%)$ \\
\hline 23. & Hypothyroidism & 24. & $6(3.4 \%)$ \\
\hline 25. & Electrocution & 26. & $2(1.1 \%)$ \\
\hline & & & \\
\hline
\end{tabular}

Table-2: Mortality in hypernatremia

\begin{tabular}{|ll|ll|}
\hline 27. & Disease condition & 28. & Mortality \\
\hline 29. & Head injury & 30. & 8 \\
\hline 31. & Sepsis & 32. & 7 \\
\hline 33. & Kidney diseases & 34. & 4 \\
\hline 35. & Cardiac disease with Diabetes mellitus, Hypertension & 36. & 2 \\
\hline
\end{tabular}

\section{Discussion}

Hypernatremia can be a life-threatening electrolyte imbalance if uncontrolled, especially in patients who have associated serious comorbid medical conditions. Long CA et.al, reported an incidence of 0.3 percent for hypernatremia in an adult in-patient British population [2]. However varying incidence has been reported by multiple authors, ranging from $0.3 \%$ to $8.9 \%$ in hospitalized adults [9]. Patients admitted to intensive care units have a higher prevalence rate of hypernatremia developing (2-9\%) and this has been attributed to a variety of common factors: loss of free water (insensible perspiration, renal and gastrointestinal tract losses) or inadequate free water intake (altered consciousness, fever, loss of thirst sensation) [3,10].

However, studies on hypernatremia in the Indian population are few and more focused on the geriatric population. Maitra $\mathrm{T}$ et.al, in one Indian study found hypernatremia to be present in $6 \%$ of in patients over 60 years and $3.5 \%$ below the age of 60 [11]. These authors also observed that though hyponatremia is the most common electrolyte abnormality encountered, elderly patients have a greater propensity to develop abnormalities in more than one electrolyte and larger studies were needed to throw more light on the exact pattern of electrolyte abnormalities in hospitalized Indian patients. In our study we found a prevalence rate of $3.14 \%$ that was much higher than studies done in general hospitals [2] as ours was a tertiary care referral center and a large number of cases were referred to our center after being unsuccessfully treated elsewhere.

Different authors have reported different disease conditions as a major primary cause for hypernatremia in general in-patient population. Snyder et.al, reported 'complications of surgery' as a major cause of hypernatremia [12], while Long CA et al reported 'febrile illness' as a major disease state developing hypernatremia in young patients [2]. In our study, head injuries were found to be a major cause of hypernatremia 37 (21.1\%). Our center overlooks one of the major highways in Kerala and most cases of road traffic accidents with head injuries that occurred on these highways were directly brought in for management: this factor besides others may have accounted for the head injury group to be a major contributor of hypernatremia. Patients with severe head injury have a high risk of developing hypernatremia due to a multitude of factors: reduced free water intake, 


\section{Original Research Article}

altered sensorium and thirst, high insensible water losses, and possibility of central diabetes insipidus (CDI) [13]. Some of the head injury patients received intravenous hypertonic fluid administration like mannitol to reduce intracranial pressure and cerebral edema, subsequently developing hypernatremia by osmotic diuresis. In one study done on patients with subarachnoid hemorrhage receiving hypertonic saline therapy, it was seen that for each $1 \mathrm{mEq} / \mathrm{L}$ increase in the running maximum daily serum sodium rate, the risk of developing acute kidney injury increased by $5.4 \%$, and the risk of death was more than twofold greater for patients who developed acute kidney injury [14]. Some centers advocate the use of hypertonic saline solution to reduce cerebral edema and this can lead to severe adverse effects through volume expansion, heart failure and hyperchloremic acidosis and hypernatremia [15].

Diabetes insipidus which may sometimes occur in head trauma $(18 \%)$ can result in hypernatremia due to pathologic polydipsia ( $>2 \mathrm{~L} / \mathrm{m} 2 / \mathrm{d})$ and polyuria. It has been shown that these head trauma patients have a three-time higher mortality than those without hypernatremia [15, 16, 17]. In our study we had four patients with DI due to head trauma and two of them died. Other authors have also reported higher incidences of hypernatremia as we have reported in neurological and neurosurgical cases (24.3- 51.5\%) [17].

While several risk factors exist for hypernatremia, the greatest risk factor is the patient's age when more than 65 years [11]. In our study the geriatric group constituted about $32.55 \%$ of the total 175 patients. Geriatric patients are unfortunately bound by a number of risk factors that lead them to develop hypernatremia: often unable to obtain oral fluids themselves independently, difficult recognition of dehydration in elderly, poor communication, need for constant monitoring with delicately balanced homeostatic mechanisms $[11,18]$. Hypernatremia in children is often due to gastrointestinal losses where more free water is lost than the electrolytes sodium and potassium which results in higher plasma sodium levels.

There were only 3 pediatric patients in the study group of which two had fever and diarrhea and one had urinary tract infection. Breast feeding-associated neonatal hypernatremia though not seen in our study patients, has been reported in infants possibly due to high breast milk sodium content which is believed to result from dehydration and malnutrition from low breast milk production [19]. In our studies 33\% were geriatric patients and $1.7 \%$ were pediatric; the rest
$(65.3 \%)$ were all general adult population indicating that even non-geriatric adult population are prone to develop hypernatremia when afflicted by disease process and should be paid attention to when admitted as in-patients. 17 of our patients had transient hypernatremia. This is often seen in severe exertion or seizure activity and is said to be due to shift of free water from extracellular to the intracellular space due to breakdown of glycogen into smaller molecules of lactate which cause increase in osmolytes [6]. Incidentally a large number of our patients in the transient hypernatremia group were involved in road traffic accidents and suffered from multiple fractures where the possibility of severe exertion and stress prevailed.

There were 23 patients with respiratory disease having hypernatremia in our study and of these, patients with pneumonia had the higher occurrences of hypernatremia, the development of which could be attributed the presence of hyperventilation, free water losses, inadequate replacement of free water, sepsis, besides other factors [20]. Septic patients are known to develop hypernatremia due to multitude of factors. 17 of patients who had hypernatremia had sepsis. In a study on burn intensive care patients carried out by the first author, an incidence of $34 \%$ of hypernatremia developing in septic patients was reported.

These study patients had higher burn area, inhalation injury and severe sepsis and were additionally incapacitated adequate free water [21]. Our center did not have a burn intensive care unit and none of the patients with burns that were admitted as in-patients developed hypernatremia as they had minor to moderate uncomplicated burns. Schwarz C et.al, in a study on 9012 patients in the department emergency medicine found that while $10 \%$ of patients had hyponatremia and $1 \%$ patients had hypernatremia, hyponatraemia was present in $14 \%$ of patients with high TSH [22].

Essential hypernatremia was also reported to have occurred in a patient with primary hypothyroidism and histiocytosis X [23]. In our study there were six patients with hypothyroidism who developed hypernatremia and the exact mechanism of their development could not be explained. Elderly patients exposed to psychotropic agents, like lithium, antipsychotics and antidepressants have been found to be at risk of developing hypernatremia from associated nephrogenic diabetes insipidus [24]. In our study group, 10(5.7\%) patients receiving psychiatric medications developed hypernatremia. We believe that incapacitation and loss of free 


\section{Original Research Article}

will to ask for free water may have additionally contributed to the hypernatremia developing. Patients with chronic renal disease tend to have multiple co morbidities that could predispose to hypernatremia [25]. In our study we had $15(8.5 \%)$ patients with hypernatremia who had underlying kidney disease.

The ubiquitous nature of hypernatremia can be seen by the fact that even pregnant and post partum women may develop hypernatremia due to a multitude of factors: fever, inadequate free water intake, gestational diabetes insipidus (GDI), reduced hepatic breakdown of vasopressinase, central adypsic hypernatremia with hypothalamic dysfunction and even increased aminopeptidase activity resulting in deficiency of ADH [4]. These authors also reported that hypernatremic post partum patients can develop 'osmotic demyelination syndrome' that manifests by encephalopathy, rhabdomyolysis, quadreparesis and white matter hyperintensities on MRI. The present study did not reveal any cases of pregnancy or postpartum patients developing hypernatremia.

Accurate diagnosis of the cause of hypernatremia will help to manage it better. Comparing the urine osmolality (Uosm) with the plasma osmolality (Posm) can help in identifying the cause of hypernatremia. While Uosm less than Posm suggests a urine concentrating defect (urine osmolality $<700 \mathrm{mOsm} / \mathrm{kg}$ ), Uosm greater than Posm suggest an intact urinary concentrating mechanism and is likely to have extra renal hypernatremia causes [26]. Correction of hypernatremia needs to be done in a slow controlled fashion and $10 \mathrm{meq} / \mathrm{L}$ in 24 hours is an accepted correction particularly in chronic hypernatremia since homeostatic mechanisms increase the intracellular concentration of ideogenic molecules that counter extracellular hyperosmolarity [4]. While there are formulas to calculate the amount of free water required to lower the serum sodium levels below $145 \mathrm{meq} / \mathrm{l}$, they fail to discuss strategies to calculate free water intake when serum $\mathrm{Na}$ is between 140 and $145 \mathrm{meq} / 1$ [27].

This study also demonstrates the fact that treating physicians tend to approach and treat hypernatremia in an empirical fashion during its onset and only when hypernatremia seems to be non-responding, specific investigations are ordered to find out the exact cause of hypernatremia and treat it in a more scientific manner.

Therefore the authors feel that a protocol needs to be established for management of hypernatremia so that early reduction in sodium levels can be achieved and complications of hypernatremia can be avoided. It has been found that hospitalized patients with hypernatremia have a higher mortality rate when compared with hospitalized patients with normal serum sodium levels [1, 28]. Hypernatremia was also found to be associated with increased perioperative morbidity and mortality with higher odds for venous thrombosis, pneumonia and major cardiac events [29]. Since many of the causes that lead to hypernatremia in hospitalized patients are based on early identification of predisposing factors and nursing support systems it has been suggested that hypernatremia be used as an indicator of quality of care in ICU settings [21, 30]. The authors believe that it is of prime importance to create a greater awareness among medical and nursing staff to pre-tag these patients and pay special interest to their fluid requirements. This paper being the first of its kind in a south Indian population should also serve to be an eye opener in the prevention and management of hypernatremic patients.

\section{Conclusion}

The rise in serum sodium leading to hypernatremia is often a preventable complication in patients admitted to a tertiary care center. Our studies reveal that treating physicians often focus on managing the developed hypernatremia in patients though it would be in the interest of patients to also focus and tag those patients who are likely to develop hypernatremia and take steps to prevent it from developing.

Hypernatremia can influence the prognosis negatively and therefore all attempts must be made to prevent hypernatremia by regular observance and prompt corrections. Geriatric patients are especially prone to develop hypernatremia and need to be specifically tagged and monitored. Pre-tagging and prompt preventive measures to avoid hypernatremia from developing will decrease hospitalization time and increase patient care cost-benefit ratio.

Hypernatremia could also be used as an indicator of quality of care and support mechanisms especially in hospitalized patients who are unable to take care of themselves. Since this was a retrospective study and patients were managed by different consultants from different departments we could not find adequate laboratory data to intricately study the pathology of hypernatremia in different disease conditions and compare them. While the first and second authors were actively involved in researching, analyzing and writing up the paper, the third and forth authors helped in collecting and compiling all the data. 


\section{Original Research Article}

What this study adds to existing knowledge? This study exposes the lack of a protocol for pre-tagging patients that are likely to develop hypernatremia as well as points to a need for a protocol management of hypernatremic patients. This paper also bares the fact that even normal adults under adverse disease conditions can develop hypernatremia like geriatric patients and they should also be keep under close observation.

In addition, there appears to be a need for further studies in this subject so that patients with hypernatremia can be better managed.

\section{Funding: Nil, Conflict of interest: None Permission of IRB: Yes}

\section{References}

1. Maggiore U, Picetti E, Antonucci E, Parenti E, Regolisti G, Mergoni M, Vezzani A, Cabassi A, Fiaccadori E. The relation between the incidence of hypernatremia and mortality in patients with severe traumatic brain injury. Crit Care. 2009; 13(4): R110. doi: 10.1186/cc7953. Epub 2009 Jul 7.

2. Long CA, Marin P, Bayer AJ, Shetty HG, Pathy MS. Hypernatraemia in an adult in-patient population. Postgrad Med J. 1991 Jul;67(789):643-5.

3. Kumar S, Guleria TC, Nand L, Gupta D, Thakur M. Serum sodium abnormality profile in hospitalized elderly patients in a tertiary care centre from north India: a cross sectional observational study. Int $\mathrm{J}$ Res Med Sci 2018; 6(1):321-327

4. Saroja AO, Naik KR, Mali RV, Kunam SR. 'Wine Glass' sign in recurrent postpartum hypernatremic osmotic cerebral demyelination. Ann Indian Acad Neurol 2013 Jan; 16 (1): 106-10. doi: 10.4103/09722327.107719 .

5. Darmon M, Timsit JF, Francais A, Nguile-Makao M, Adrie C, Cohen Y, Garrouste-Orgeas M, GoldgranToledano D, Dumenil AS, Jamali S, Cheval C, Allaouchiche B, Souweine B, Azoulay E. Association between hypernatraemia acquired in the ICU and mortality: a cohort study. Nephrol Dial Transplant. 2010 Aug; 25(8):2510-5. doi: 10.1093/ndt/gfq 067. Epub 2010 Feb 17.

6. Rose BD. Clinical physiology of acid-base and electrolyte disorders 5thed. New York, NY: McgrawHill; 352:220-228.
7. Soupart A, Penninckx R, Namias B, Stenuit A, Perier O, Decaux G. Brain myelinolysis following hypernatremia in rats. J Neuropathol Exp Neurol. 1996 Jan; 55 (1):106-13.

8. Beck LH. The aging kidney. Defending a delicate balance of fluid and electrolytes. Geriatrics. 2000 Apr; 55 (4):26-8, 31-2.

9. Shea AM, Hammill BG, Curtis LH, Szczech LA, Schulman KA. Medical costs of abnormal serum sodium levels. J Am Soc Nephrol. 2008 Apr; 19(4):76470. doi: 10.1681/ASN.2007070752.

10. Lindner G, Funk GC, Schwarz C, Kneidinger N, Kaider A, Schneeweiss B, Kramer L, Druml W. Hypernatremia in the critically ill is an independent risk factor for mortality. Am J Kidney Dis. 2007 Dec; 50 (6): 952-7.

11. Maitra T, Thakuria R, Basumatary A. Dyselectrolytemia in elderly: A hospital based study. J Evid Based Med Healthc. 2016; 3(30), 1348-51. doi: 10. 18410/ jebmh/2016/308.

12. Snyder NA, Feigal DW, Arieff AI. Hypernatremia in elderly patients. A heterogeneous, morbid, and iatrogenic entity. Ann Intern Med. 1987 Sep;107(3): 309-19.

13. Tisdall M, Crocker M, Watkiss J, Smith M. Disturbances of sodium in critically ill adult neurologic patients: a clinical review. J Neurosurg Anesthesiol. 2006 Jan; 18(1):57-63.

14. Kumar AB, Shi Y, Shotwell MS, Richards J, Ehrenfeld JM. Hypernatremia is a significant risk factor for acute kidney injury after subarachnoid hemorrhage: a retrospective analysis. Neurocrit Care. 2015 Apr; 22 (2) : 184-91. doi: 10. 1007 / s 12028- 0140067-8.

15. Li M, Hu YH, Chen G. Hypernatremia severity and the risk of death after traumatic brain injury. Injury. 2013 Sep; 44(9):1213-8. doi: 10.1016/j.injury. 2012. 05.021 .

16. Hadjizacharia $\mathrm{P}$, Beale EO, Inaba $\mathrm{K}$, Chan LS, Demetriades D. Acute diabetes insipidus in severe head injury: a prospective study. J Am CollSurg. 2008 Oct; 207 (4): 477-84.doi: .1016/j. jamcollsurg. 2008. 04.017 . 


\section{Original Research Article}

17. Paiva WS, Bezerra DA, Amorim RL, Figueiredo EG, Tavares WM, De Andrade AF, Teixeira MJ. Serum sodium disorders in patients with traumatic brain injury. Ther Clin Risk Manag. 2011; 7:345-9. doi: 10.2147/ TCRM.S17692. Epub 2011 Aug 11.

18. Aiyagari V, Deibert E, Diringer MN. Hypernatremia in the neurologic intensive care unit: how high is too high? J Crit Care. 2006 Jun; 21(2):163-72.

19. Mujawar NS, Jaiswal AN. Hypernatremia in the Neonate: Neonatal Hypernatremia and Hypernatremic Dehydration in Neonates Receiving Exclusive Breast feeding. Indian Journal of Critical Care Medicine : 2017; 21(1):30-33.

20. VAN DE Louw A, Shaffer C, Schaefer E. Early intensive care unit-acquired hypernatremia in severe sepsis patients receiving $0.9 \%$ saline fluid resuscitation. Acta Anaesthesiol Scand. 2014 Sep; 58 (8):1007-14. doi: 10.1111/aas.12368. Epub 2014 Jul 15.

21. Ebrahim MK, George A, Bang RL. Only some septicaemic patients develop hypernatremia in the burn intensive care unit: why? Burns. 2002 Sep;28(6):543-7.

22. Schwarz C, Leichtle AB, Arampatzis S, Fiedler GM, Zimmermann H, Exadaktylos AK, Lindner G. Thyroid function and serum electrolytes: does an association really exist? Swiss Med Wkly. 2012 Sep 17; 142: w13669. doi: 10.4414/smw.2012.13669.

23. Ma JT, Ho FC, Wang C, Lam KS, Yeung RT. Primary hypothyroidism and essential hypernatremia in a patient with histiocytosis X. Aust N Z J Med. 1985 Feb; 15(1):72-4.
24. Sajadi S, Yu C, Sylvestre JD, Looper KJ, Segal M, Rej S. Does lower urine-specific gravity predict decline in renal function and hypernatremia in older adults exposed to psychotropic medications? An exploratory analysis. Clin Kidney J. 2016 Apr; 9 (2):268-72. doi: 10. 1093/ckj/sfv132. Epub 2015 Dec 10.

25. Kovesdy CP. Significance of hypo- and hypernatremia in chronic kidney disease. Nephrol Dial Transplant. 2012 Mar; 27(3):891-8. doi: 10.1093/ndt/ gfs038.

26. Latcha S, Lubetzky M, Weinstein AM. Severe hyperosmolarity and hypernatremia in an adipsic young woman. Clin Nephrol. 2011 Nov; 76(5):407-11.

27. Varun S, Bhaskar E, Abraham G, Arunkumar AS, Renuka MK. Risk factors for hospital-acquired hypernatremia among critically ill medical patients in a setting utilizing a preventive free water protocol: Do we need to do more? Indian J Crit Care Med. 2013 Jan; 17 (1):28-33. doi: 10.4103/0972-5229.112157.

28. Palevsky PM, Bhagrath R, Greenberg A. Hypernatremia in hospitalized patients. Ann Intern Med. 1996 Jan 15;124(2):197-203.

29. Leung AA, McAlister FA, Finlayson SR, Bates DW. Preoperative hypernatremia predicts increased perioperative morbidity and mortality. Am J Med. 2013 Oct;126(10):877-86. doi:10.1016/j.amjmed. 2013.02. 039. Epub 2013 Aug 1.

30. Polderman KH, Schreuder WO, Strack van Schijndel RJ, Thijs LG. Hypernatremia in the intensive care unit: an indicator of quality of care? Crit Care Med. 1999 Jun;27(6):1105-8.

\section{How to cite this article?}

George A, Alexander R, Abraham S.T, Rhutu Venugopal E V. Profile of Hypernatremia in a Tertiary care hospital : A review of 175 cases over a 5 year period in a South Indian population. Int J Med Res Rev 2018;6 (03):159-165. doi:10.17511/ijmrr. 2018.i03.05. 